\title{
Limitaciones y barreras en el uso del condón en jóvenes universitarios de Cali, Colombia
}

\author{
Limitations and barriers for condom use in college students from Cali, Colombia
}

\author{
Claudia Patricia Valencia-Molina1* orcid.org/0000-0002-9558-4146 \\ Delia Concepción Burgos-Dávila' orcid.org/0000-0002-4373-6427 \\ Martha Cecilia Sabala-Moreno² orcid.org/0000-0003-3876-7226 \\ Álvaro José Sierra-Perez1,3 orcid.org/0000-0002-4903-3060
}

1. Escuela de Enfermería, Universidad del Valle. Cali, Colombia

2. Programa de Psicología, Universidad del Valle. Palmira, Colombia

3. Grupo PROMESA, Universidad del Valle. Cali, Colombia

\section{Resumen}

Introducción: El condón es un método de prevención de las Infecciones de Transmisión Sexual (ITS) y del embarazo, sin embargo, su uso en jóvenes colombianos es bajo. Objetivo: Identificar las barreras y limitaciones individuales, culturales e institucionales, en el uso del condón en jóvenes universitarios. Materiales y métodos: Investigación cualitativa, con 4 grupos focales mixtos con jóvenes de 17 a 24 años. Se realizó codificación con el software ATLAS.TI y un análisis temático. Resultados: A nivel individual se evidencia baja intención de uso, la confianza en la pareja aleja el temor de una ITS y el embarazo es la principal preocupación que desplaza su uso por otro método anticonceptivo. A nivel cultural las relaciones de género refuerzan que la mujer es responsable de los anticonceptivos y el hombre del condón. A nivel institucional, las políticas y los servicios de salud limitan su acceso. La lógica mercantilista y el enfoque de riesgo limitan la distribución gratuita y masiva. Conclusiones: El reconocimiento de factores individuales y sociales que limitan el uso del condón, permite la promoción del cuidado de sí y del otro; además la orientación de estrategias para promover una adecuada atención en los servicios de Salud Sexual y Reproductiva.

Palabras clave: Condón; adulto joven; sexualidad; ITS; servicios de salud. (Fuente: DeCS, Bireme).

\begin{abstract}
Introduction: Condom is a method to prevent Sexually Transmitted Diseases (STDs) and pregnancy. However, the use of condom by the young Colombian population is low. Objective: To identify individual, cultural, and institutional barriers and limitations of condom use in university students. Materials and methods: A qualitative research was developed with 4 mixed focus groups of young people aged from 17 to 24 years. Coding was carried out with ATLAS.TI together with a thematic analysis. Results: At the individual level, it was observed: a low intention of condom use; trust in partner that reduces the fear of STDs; and pregnancy is the main reason why condom is replaced by a different contraceptive method. At the cultural level, gender relationships reinforce the view that women and men are responsible for the use of contraceptives and condoms, respectively. At the institutional level, health policies and services restrict the access to condoms. Mercantilism logic and risk approach limit free and massive distribution of condoms. Conclusions: The recognition of individual and social factors that limit the use of condoms facilitates the promotion of care for one self and for others as well as the coordination of strategies to promote adequate sexual and reproductive health services.
\end{abstract}

Keywords: Condoms; young adult; sexuality; sexually transmitted diseases; health services. (Fuente: DeCS, Bireme).

\footnotetext{
*Autor de correspondencia

Claudia Patricia Valencia Molina

e-mail: claudia.p.valencia@correounivalle.edu.co
} 


\section{Introducción}

Según datos de la Organización Mundial de la Salud (OMS) a enero del 2020, en el mundo alrededor de 16 millones de adolescentes entre los 15 y 19 años, y 1 millón de adolescentes menores de 15 años ha dado a luz cada año(1), en ese sentido, la OMS refiere qué si bien ha habido una reducción en las tasas de embarazo en la adolescencia desde 1990 al 2019, aún se presenta en un $11 \%$ de todos los nacimientos en mujeres de 15 a 19 años, donde el 95\% de ellos ocurre en países de ingresos bajos y medios, lo que genera, como problemática mundial, complicaciones durante la gestación y el parto, que se considera la segunda causa de muerte en esta población. Cada año, alrededor de 3 millones de mujeres en ese rango de edad se realizan un aborto exponiendo su salud a altos riesgos(2).

Según el Departamento Nacional de Planeación de Colombia, en el año 1990 la cifra para los embarazos adolescentes estaba en el 12,8\%, aumentando paulatinamente a través de los años al $20,5 \%$ en el 2005. Para el 2010 se muestra el primer descenso a $19,5 \%$ y al 2014 la cifra que se tiene es de 17,5\%(3). Esta problemática en el país mostró sus picos de ascenso entre los años 1990 y 2005, pero ha logrado su descenso en los últimos 9 años.

En relación con el Virus de la Inmunodeficiencia Humana (VIH), El Programa Conjunto de las Naciones Unidas sobre el VIH/Sida (ONUSIDA) reporta al 2019, que alrededor de 37,9 millones de personas en el mundo conviven con el virus, de los cuales 1,7 millones se corresponden a niños menores de 15 años, asimismo, se presentaron 1,7 millones de casos nuevos en el mundo, sin embargo, se considera que desde el 2010 los casos nuevos han descendido en un $16 \%$ al $2018^{(4)}$. Para Colombia, ONUSIDA reporta que al 2018 alrededor de 160.000 personas conviven con el virus, de los cuales 3.600 corresponden a menores de 14 años(5). Por su parte, el VII estudio centinela, reporta que la prevalencia del VIH fue estimada para las personas entre 15 y 49 años en $0,22 \%$; la epidemia sigue estando concentrada en grupos más vulnerables con una prevalencia por encima del 5\%, como en el caso de los hombres que tienen sexo con hombres (HSH) entre quienes se ha reportado cifras entre el $10,18 \%$ y el $24 \%{ }^{(3)}$.

A lo largo de la epidemia la preocupación por generar estrategias de prevención ha sido una de las prioridades de investigadores y organizaciones de la salud, muchos de ellos orientados a la identificación de los factores que protegen o exponen al riesgo a la población, cuyos resultados deben ser considerados para el diseño de programas de prevención. Uno de los métodos más efectivos para reducir la transmisión del virus del VIH y prevenir los embarazos ha sido el condón(6,7).

Un estudio realizado en Cali, con una muestra de 1.704 jóvenes de universidades públicas y privadas, mostró que la proporción de jóvenes que reportan utilizar "siempre" el condón en sus encuentros sexuales fue sólo del $31,5 \%$, cifra que se mantiene estable desde el año 2008 en el cual se aplicó la misma encuesta(8). Este porcentaje es muy bajo para población joven sexualmente activa, comparado con otros países como Argentina y España cuyos porcentajes son iguales o mayores al $80 \%$ o moderado versus las cifras de otros países latinoamericanos como Cuba, en donde los datos no superan el $21,3 \%{ }^{(9)}$. Las diferencias por género muestran en el estudio de Canaval y Valencia del 2008, que los hombres reportaron su uso "siempre" en mayor proporción (36\%) que las mujeres (27\%) $(p<0,001)$. Por su parte, entre la población no heterosexual su uso también fue menor (23\%) que entre los heterosexuales (32\%) $(p=0,029)$.

A raíz de este diagnóstico y después de analizar que la cifra del uso del condón se mantiene estable y en niveles considerablemente bajos comparada con poblaciones jóvenes de otros países del mundo y de Latinoamérica, se identificó la necesidad de plantear una investigación denominada proyecto PRESERVA, para diseñar, implementar y evaluar una intervención estructural que permitiera abordar las barreras de orden individual, intermedio y distal que limitan el uso del condón en jóvenes universitarios de Cali, Colombia. En el desarrollo de dicha investigación, se planteó la realización de una primera fase cualitativa cuyo objetivo fue identificar las barreras y limitaciones individuales, culturales e institucionales, en el uso consistente del condón en la población sujeto de estudio. A través de este artículo se presentan los resultados de esta primera fase del proyecto.

\section{Materiales y métodos}

Se desarrollaron en el municipio de Santiago de Cali, Colombia, cuatro grupos focales, entre los meses de octubre y noviembre del 2017; tres grupos se 
conformaron con jóvenes pertenecientes a una universidad pública donde se realizaría la intervención PRESERVA, y el cuarto grupo se conformó con jóvenes multiplicadores en salud sexual y salud reproductiva (SSR) vinculados a la Secretaría de Salud Pública Municipal que realizan labores de sensibilización y educación por pares en los diferentes barrios de la ciudad. Se escogió la técnica de los grupos focales dado que están más indicados para examinar cómo se desarrollan y operan las ideas en un determinado contexto cultural(10).

Los criterios de selección de los participantes fueron mujeres y hombres entre los 15 y los 24 años, con vida sexual activa, inactiva o sin iniciación. Se escogieron estos dos grupos de jóvenes dadas sus diferencias de acuerdo con sus experiencias, expectativas y trayectoria en el tema. Los jóvenes pares en SSR, se diferencian de los jóvenes universitarios en ostentar un cúmulo de conocimientos en el tema de la salud sexual y la salud reproductiva a partir de las capacitaciones que han recibido para ejercer como par en las comunas de la ciudad. Asimismo, por tener una mirada más aproximada de las limitaciones en el uso consistente del condón desde su propia experiencia y de los discursos y razones expresados por los jóvenes, padres y maestros con quienes han interactuado en su práctica como pares educadores. Se empleó como instrumento una guía de entrevista con preguntas para estimular la participación equitativa entre los asistentes(10).

\section{Análisis de datos}

Los datos fueron codificados a través del software ATLAS.TI. Para reducir posibles sesgos a la hora de analizar los datos, se usó la estrategia de Análisis Cualitativo Consensuado (Consensual Qualitative Research, CQR), que opera bajo la lógica de generar consenso entre los investigadores a la hora de la codificación de los datos, para reducir los posibles sesgos de los mismos y captar de manera sustancial la complejidad de los datos(11).

Se realizó un análisis temático que permitió identificar, segmentar y establecer las ideas principales de los participantes. En primer lugar, la calidad de las transcripciones fue revisada para después organizar los datos en categorías principales alrededor de las limitaciones en el uso consistente del condón, desde el plano proximal e intermedio y las barreras desde el nivel estructural.
Estas categorías fueron: experiencia en el uso del condón; representaciones sociales - contexto y acceso al condón. Todas las categorías se analizaron con enfoque de género y diferencial. Una vez procesada y analizada la información se hizo la devolución al grupo de jóvenes para validar su veracidad. Esto como una estrategia para conservar el rigor científico (principio de credibilidad), y a su vez para realimentarlos con las conclusiones del trabajo.

\section{Consideraciones éticas}

Se obtuvo aval del Comité de Ética de la Facultad de Salud de la Universidad del Valle, Cali, Colombia. Se aplicó un consentimiento informado mediante el cual se explicó el objetivo de la actividad, los riesgos y beneficios de participar en la misma. Así mismo se hizo énfasis en reiterar su participación libre y voluntaria. Durante el desarrollo del grupo focal se solicitó a los participantes asignarse un pseudónimo para garantizar su confidencialidad durante el desarrollo de este, así como en la publicación de sus resultados.

\section{Resultados}

La población participante estuvo conformada por 38 jóvenes, distribuidos entre 19 hombres y 19 mujeres con edades entre los 15 y 24 años. La mayoría (27) se asumieron con una orientación heterosexual (Tabla 1).

Tabla 1. Características sociodemográficas de los jóvenes participantes

\begin{tabular}{lrrr}
\hline \multicolumn{1}{c}{ Categoría } & $\begin{array}{c}\text { Grupo focal } \\
\text { jóvenes } \\
\text { universitarios }\end{array}$ & $\begin{array}{c}\text { Grupo focal } \\
\text { pares en SSR }\end{array}$ & Total \\
\hline Género & 9 & 10 & 19 \\
Hombres & 12 & 7 & 19 \\
Mujeres & 21 & 17 & 38 \\
Total & & & \\
Orientación sexual & 4 & 4 & 8 \\
Homosexual & 1 & 2 & 3 \\
Bisexual & 14 & 13 & 27 \\
Heterosexual & & & \\
\hline
\end{tabular}

\section{A. Experiencia en el uso del condón}

Los jóvenes tienen claro que las principales razones para el uso de condón es la prevención del embarazo y las ITS como lo muestra el siguiente relato: "Pienso del condón que es un buen método no sólo anticonceptivo sino también para prevenir las enfermedades de transmisión sexual y pues algunas 
personas prefieren no utilizarlo, pero sin embargo creo que es muy necesario"(Cris, 20a).

Sin embargo, la instauración de una vida sexual activa, la estabilidad que va adquiriendo la relación y el consiguiente incremento de la confianza en la pareja, aleja el temor a una infección de transmisión sexual y mantiene la prioridad del embarazo como la principal preocupación.

Otras razones no menos importantes, se relacionan con la baja cultura de la prevención, que se refleja en la baja intención de uso. Ninguno de los jóvenes participantes del grupo focal cargaba uno en ese momento, argumentando: "para que voy a traer condones a la universidad" (Jack, 21a).

También existen argumentos de orden individual que están relacionados con la idea del amor romántico en la cual omitir el uso del condón representa una compenetración amorosa e incluirlo es muestra de desconfianza. Igualmente, persiste la clasificación que hacen los jóvenes de sus parejas en razón a su apariencia (pura e impura), familiaridad (conocida o desconocida), lo cual define el riesgo de tener una relación sexual desprotegida: "Si es una pareja eventual con la que me veo frecuentemente y ella planifica, no utilizo condón. Si alguien que apenas conocí, ahí si uso el condón"(Valeri, 24a).

Llamó poderosamente la atención la vigencia de estereotipos patriarcales en los cuales algunos hombres definen la calidad del condón en función de la apariencia de su pareja; esto también evidencia el desconocimiento y el valor del condón para el cuidado de sí y del otro. "Pues si yo me voy a acostar con una niña "bien" escojo el mejor condón, pero si es una chica " $X$ " me consigo uno de los baratos"(Paco,22a).

\section{B. Representaciones sociales y contexto}

Los relatos de los jóvenes muestran que aún persisten algunas diferencias por género en las razones de uso y en el acceso. Se mencionó la baja capacidad de agencia de la mujer para negociar el uso del condón y la vergüenza para adquirirlo: "algunas mujeres son muy sumisas, les da pena o miedo, no le dicen al hombre que utilice condón si no, que se quedan calladas, no dicen nada y prefieren tener la relación así"(Jacinta, 19a).

Las mujeres lesbianas identifican las mayores limitaciones en el acceso a la información y a métodos de protección en relaciones homosexuales femeninas, al relatar que nunca o en contadas ocasiones escuchan hablar acerca de la necesidad de usar barreras de protección; en cambio les insisten en el uso de anticonceptivos sin indagar primero su orientación sexual. Los jóvenes pares educadores en SSR durante su trabajo con las diferentes comunidades (padres, maestros y jóvenes) resaltan la vigencia de preceptos culturales y las normas de crianza que desestimulan el uso consistente del condón en los jóvenes. "También hay que considerar la parte machista del país, porque a mí me pasó que estaba hablando con un profesor y llegó otro a meter ruido diciendo: "si yo llego a ver a mi hija con un condón, la saco a pata de la casa" imagínense, siendo un profesor" (Tomy, 23a).

Entre los maestros se evidencia la influencia de sus creencias religiosas, al considerar que hablar de sexualidad, de métodos de anticoncepción o de protección, induce o incrementa las relaciones sexuales.

\section{Acceso al condón}

Múltiples barreras de acceso al condón se identificaron en la investigación. Algunas relacionadas con los preceptos culturales que señalan a la mujer cuando va a la droguería a conseguirlos; otros con cuestiones económicas y la más importante con la restricción que imponen los servicios de salud en la provisión a los jóvenes. "En días pasados fui a pedir condones a mi EPS contributiva y ellos allá me dijeron que no me daban condones porque ya me habían dado un método anticonceptivo, que es que ellos solo entregaban un método anticonceptivo por persona; yo les dije que trabajaba en los servicios de salud sexual, que ese era mi derecho, entonces me dijeron que ellos sabían cuáles eran mis derechos pero eso eran normas de la empresa; que solo entregaban un método"(Vicky, 23a).

\section{Discusión}

La población participante de esta investigación estuvo representada en igual proporción por hombres y mujeres. La posibilidad de escuchar los relatos de los jóvenes a quien va dirigida la intervención, quienes evidencian poca información y a los jóvenes educadores en SSR fue muy valiosa, dado que permitió reconocer aspectos individuales, sociales, culturales e institucionales variados. En relación con la experiencia en el uso del condón, esta 
investigación ratifica los hallazgos de otras similares, en la cuales el embarazo es la principal preocupación cuando sostienen relaciones sexuales penetrativas. Por ello, en la medida que la relación se va formalizando, las parejas adoptan otro método anticonceptivo que les brinde mayor seguridad desplazando el uso del condón(13-16).

La vigencia de estereotipos patriarcales que definen el uso y calidad de la protección de acuerdo a la apariencia de la pareja sexual, mantienen en el imaginario la dualidad entre la pareja bien, limpia, de la casa vs. la pareja libertina, abierta, sucia, lo cual ha sido documentado por otros autores a nivel local( 7,17$)$ e internacional(18-20).

Los resultados del estudio evidencian como lo han hecho otros ${ }^{(16,21)}$, la persistencia de desigualdades en razón al género que limitan la intención y el uso de medidas protectoras en la vivencia de la sexualidad, como la vergüenza al adquirirlos y la baja agencia para negociar con la pareja. Llama la atención que esta percepción es manifiesta por las mujeres y evidenciada también por los hombres, en ese sentido, estudios en Latinoamérica muestran que las diferencias de género limitan la atención en salud sexual de forma enfocada a las necesidades del joven(22).

Las limitaciones identificadas por algunas mujeres lesbianas del estudio, en el acceso a la información y a métodos de protección en relaciones homosexuales femeninas, ha sido considerado como otra forma de maltrato en lo social en especial en los servicios de salud(23,24). Algunos estudios reportan la poca difusión de la necesidad de la protección en el marco de las relaciones lésbicas, así como de los métodos o estrategias efectivas ${ }^{(25) .}$

Tanto hombres como mujeres perciben insuficientes programas de consejería en sexualidad, una orientación hetero hegemónica y limitados recursos en los servicios de salud para orientar una sexualidad protegida, factores que se relacionan con otros hallazgos de estudios en otros países(26,27). En el informe sobre Diversidad Sexual, Discriminación y Pobreza frente al acceso a la Salud Pública en Colombia(28), los testimonios de la población homosexual femenina juzgan como deficiente la calidad de atención en salud, mientras que, desde el colectivo gay, la lucha contra la estigmatización y el VIH/Sida es una de las prioridades más destacadas.
Tanto en maestros como en padres de familia se evidencia la influencia de sus creencias religiosas y normas de crianza al considerar que hablar de sexualidad, de métodos de anticoncepción o de protección, induce o incrementa las relaciones sexuales. Esta realidad limita la posibilidad de ofrecer a los jóvenes educación, o remitir a los servicios de salud para que obtengan asesoría en SSR ${ }^{29}$. La evidencia muestra que en los países que han optado por hablar desde edades tempranas con los jóvenes acerca de la sexualidad y reconocer su vivencia en la etapa juvenil, son los que tienen las tasas más bajas de embarazos no planeados en jóvenes y adolescentes ${ }^{(30,31)}$.

Las barreras de acceso que imponen los servicios de salud en razón a la lógica mercantilista y a su enfoque de riesgo en los programas, así como el desconocimiento y la baja apropiación de los derechos sexuales y reproductivos en la población juvenil, limitan la distribución gratuita y el acceso del condón a todos los jóvenes. Otros investigadores a nivel internacional han identificado este aspecto como un factor vertebral en el no uso del condón en los jóvenes y en poblaciones vulnerables(16).

\section{Conclusiones}

Los resultados de este estudio muestran a nivel individual, las limitaciones que tienen los jóvenes con relación al uso consistente del condón, dadas por aspectos de orden cultural y creencias: uso de acuerdo al tipo de pareja, a la apariencia de esta y directamente relacionadas con el empoderamiento de la mujer, donde consideran actitudes en estas como "sumisión" que impide la negociación y exigencia del uso del condón con la pareja.

En parejas homosexuales, especialmente en mujeres, se reconoce la falta de conocimiento en relación al uso de métodos de barrera en las prácticas sexuales, donde persisten ideas de prevención del embarazo a través del uso de métodos de planificación familiar.

A nivel del sistema de salud, reconocen limitaciones que van ligadas a la accesibilidad a los servicios, y la obtención de información y asesoría profesional en temas relacionados con su salud sexual y salud reproductiva. Asimismo, la falta de difusión y promoción de los programas de SSR de los servicios de salud impacta sobre el acceso de los jóvenes. 
Se identifica limitaciones desde los profesionales en quienes persiste el enfoque en prevención del embarazo a través del uso de métodos anticonceptivos, más no se promueve el uso consistente del condón, en especial en los jóvenes universitarios. Por otro lado, reconocen factores como el desconocimiento de los profesionales en atención diferenciada y de género, en especial para población homosexual.

Se ratifica la existencia de limitantes asociados con los determinantes sociales en relación al uso consistente del condón. Estas limitaciones deben ser tenidas en cuenta en las intervenciones estructurales que busquen la promoción del condón en jóvenes universitarios. Estas no sólo deben promover habilidades para el cuidado de sí y del otro, sino además la deconstrucción de mitos y preceptos culturales, así como la distribución y acceso masivo de los recursos para la prevención.

Desde el punto de vista metodológico, la presente investigación permitió validar la posibilidad de realizar grupos focales en el tema de la sexualidad, con grupos mixtos de hombres y mujeres. Los textos de investigación cualitativa recomiendan mantener grupos homogéneos, especialmente si se tratan temas relativos a la sexualidad. Los jóvenes de esta investigación manifestaron la riqueza de las discusiones entre hombres y mujeres relativas al tema.

Agradecimientos: Los autores agradecen a los jóvenes que participaron en esta investigación por sus valiosos aportes.

Financiación: Este proyecto se realizó con el apoyo de la Vicerrectoría de Investigaciones de la Universidad del Valle, convocatoria 2017.

Conflicto de intereses: Los autores declaran no tener conflicto de intereses con los financiadores ni con ninguna entidad.

\section{Referencias}

1. Organización Mundial de la Salud. El embarazo en la adolescencia [Internet]. Centro de prensa. 2020 [cited 2020 May 12]. p. 1. Available from: https://www.who.int/es/news-room/factsheets/detail/adolescent-pregnancy

2. UNFPA. Embarazo Adolescente: Panorama [Internet]. UNFPA. 2017. p. 1. Available from: https://www.unfpa.org/es/embarazo-adolescente
3. Profamilia. Encuesta Nacional de Demografía y Salud ENDS. Bogotá, Colombia; 2015. Available from: https://profamilia.org.co/wpcontent/uploads/2018/12/ENDS-TOMO-I.pdf

4. ONUSIDA. Hoja informativa - Estadisticas Mundiales Sobre el VIH [Internet]. 2019. Available from: https://www.unaids.org/sites/default/files/media_asset/U NAIDS_FactSheet_es.pdf

5. ONUSIDA. HIV and AIDS Estimates Colombia [Internet]. $2018 . \quad$ Available from: https://www.unaids.org/es/regionscountries/countries/co lombia

6. Espada JP, Morales A, Guillén-Riquelme A, Ballester R, Orgilés M. Predicting condom use in adolescents: a test of three socio-cognitive models using a structural equation modeling approach. BMC Public Health [Internet]. 2015;16(1):35. Available from: https://doi.org/10.1186/s12889-016-2702-0

7. Uribe Alvarado JI, Carrillo Sierra SM, Riaño-Garzón ME, Zacarias Salinas X, Bautista-Sandoval M, Bonilla-Cruz N. Expresiones de la sexualidad y del uso del condón en las relaciones sexuales en jovenes universitarios de Colombia. Aportes a la clínica psicológica. Arch Venez Farmacol y Ter. 2018;35(5):456.

8. Valencia CP, Canaval GE. Factores que predisponen, facilitan y refuerzan el uso del condón en jóvenes universitarios de Cali, Colombia. Revista Salud Pública. 2012;14(5):810-21. Available from: https://www.scielosp.org/pdf/rsap/2012.v14n5/810-821

9. García Roche RG, Cortés Alfaro A, Vila Aguilera LE, Hernández Sánchez M, Mesquia Valera A. Comportamiento sexual y uso del preservativo en adolescentes y jóvenes de un área de salud. Rev Cuba Med Gen Integr [Internet]. 2006;22(1). Available from: http://scielo.sld.cu/scielo.php?script=sci_arttext\&pid=S086 4-21252006000100003\&lng=es

10. Hamui-Sutton A, Varela-Ruiz M. La técnica de grupos focales. Investigación en educación medica. 2013;2(5):55$60 . \quad$ Available from: https://www.redalyc.org/articulo.oa?id=349733230009

11. Hill CE, Knox S, Thompson BJ, Williams EN, Hess SA, Ladany N. Consensual qualitative research: An update. J Couns Psychol. 2005;52(2):196-205. Available from: https://psycnet.apa.org/record/2005-03263-009

12. Sierra Pérez ÁJ, Valencia CP, Canaval GE. Conocimientos, actitudes y prácticas en el uso del condón en jóvenes de una universidad pública del sur-occidente colombiano: un estudio comparativo 2011 - 2014. Universidad del Valle; 2016. Available from: https://bibliotecadigital.univalle.edu.co/bitstream/handle/ 10893/10086/CB-0560292.pdf?sequence=1\&isAllowed=y

13. Blanc Molina A, Rojas Tejada AJ. Uso del preservativo, número de parejas y debut sexual en jóvenes en coito vaginal, sexo oral y sexo anal. Rev Int Andrología [Internet]. 2018;16(1):8-14. Available from: https://linkinghub.elsevier.com/retrieve/pii/S1698031X17 300353

14. Canaval GE, et al. Resultados componente cuantitativo: acciones, conocimientos, actitudes y prácticas preventivas frente al VIH. En: Sevilla-Peñu, et. al,. Tramas, sexualidades y prevención del VIH en jóvenes universitarios de Cali. Santiago de Cali. Sello Editorial Javeriano y Editorial Universidad del Valle, 2019. p. 181-222. 
15. Camacho Rodríguez DE, Pabón Valera Y. Percepciones que afectan negativamente el uso del condón en universitarios de la Costa Caribe Colombiana. Hacía la Promoción la Salud. 2014;19(1):54-67. Available from: http://www.scielo.org.co/scielo.php?script=sci_arttext\&pid $=$ S0121-75772014000100005\&lng=en

16. Ministerio de salud y Protección social, UNFPA. Análisis de situación de condones en Colombia. Vol. 3, UNFPA. Bogotá, Colombia; 2015. 1-118 p.

17. Sevilla Peñuela TM. "Sexo inseguro": un análisis de la racionalidad como parte del riesgo entre jóvenes caleños y caleñas. Rev Latinoam Ciencias Soc Niñez y Juv. 2008;6(1):257-94. Available from: http://biblioteca.clacso.edu.ar/Colombia/alianza-cindeumz/20130822011607/RevistaLatinoamericanaVol.6N.1en ero-junio2008.pdf

18. Skidmore D, Hayter E. Risk and sex: Ego-centricity and sexual behaviour in young adults. Health Risk Soc. 2010;2(1):23-32. Available from: https://doi.org/10.1080/136985700111422

19. Grimberg M. Iniciación sexual, prácticas sexuales y prevención del VIH/SIDA en jóvenes de sectores populares: un análisis antropológico de género. Horizontes Antropológicos. 2002;8(17):47-75. Available from: https://doi.org/10.1590/S0104-71832002000100003

20. Francis DB, Noar SM, Widman L, Willoughby JF, Sanchez DM, Garrett KP. Perceptions of a campus-wide condom distribution programme: An exploratory study. Health Educ J [Internet]. 2016;75(8):998-1011. Available from: http://journals.sagepub.com/doi/10.1177/001789691664 8994

21. Bahamon Muñeton MJ, Vianchá Pinzón MA, Tobos Vergada AR. Prácticas y Conductas Sexuales de Riesgo en Jóvenes: una Perspectiva de Género. Psicol desde el Caribe [Internet]. 2014;31(2):327-53. Available from: http://www.scielo.org.co/pdf/psdc/v31n2/v31n2a08.pdf

22. Obach A, Sadler M, Aguayo F, Bernales M. Salud sexual y reproductiva de hombres jóvenes en Chile: resultados de un estudio cualitativo. Rev Panam Salud Pública [Internet]. 2018;42:e124. Available from: http://iris.paho.org/xmlui/handle/123456789/49568

23. Volio Monge $R$, Martínez de la Colina M. Identidades Lésbicas en Nicaragua: Trayectorias vitales y perspectivas futuras. 2013.
24. Gómez Bedoya E, Insuasti Moncayo A, Orcasita Pineda L. Servicios de salud sexual y salud reproductiva: experiencias de un grupo de mujeres lesbianas y bisexuales. Pontificia Universidad Javeriana Cali; 2018.

25. Orcasita-Pineda LT, Palma-García DM, Munevar-Cabal J. Percepción del riesgo frente al VIH: estudios en mujeres homosexuales. Revista de Psicología Universidad de Antioquia. 2013;5(2):63-79. Available from: https://revistas.udea.edu.co/index.php/psicologia/article/ view/19685

26. Silberman P, Buedo PE, Burgos LM. Barreras en la atención de la salud sexual en Argentina: percepción de las mujeres que tienen sexo con mujeres. Rev Salud Pública [Internet]. 2016;18(1):1-12. Available from: https://doi.org/10.15446/rsap.v18n1.48047

27. Alonzo J, Mann L, Simán F, Sun CJ, Andrade M, Villatoro G, et al. Perspectives to improve the sexual health of sexual and gender identity minorities in Guatemala. EHQUIDAD [Internet]. 2016;(5):51-70. Available from: https://doi.org/10.15257/ehquidad.2016.0002

28. Jaime M. Diversidad sexual, discriminación y probreza frente al acceso a la Salud Pública: demandas de la comunidad TLGBI en Bolivia, Colombia, Ecuador y Perú. 2013, CLACSO: Buenos Aires. 252 p. Available from: http://biblioteca.clacso.edu.ar/clacso/clacsocrop/20130829115928/DiversidadSexual.pdf

29. Abril Valdez E, Román Pérez R, Cubillas Rodríguez MJ, Domínguez Ibáñez SE. Creencias sobre el uso del condón en una población universitaria. Cienc ergo sum [Internet]. 2018;25(3):1-12. Available from: https://doi.org/10.30878/ces.v25n3a5

30. Oringanje C, Meremikwu MM, Eko H, Esu E, Meremikwu A, Ehiri JE. Interventions for preventing unintended pregnancies among adolescents. Cochrane Database Syst Rev [Internet]. 2016;(2). Available from: http://doi.wiley.com/10.1002/14651858.CD005215.pub3

31. Mason-Jones AJ, Sinclair D, Mathews C, Kagee A, Hillman A, Lombard C. School-based interventions for preventing HIV, sexually transmitted infections, and pregnancy in adolescents. Cochrane Database Syst Rev [Internet]. 2016;(11):94. Available from: http://doi.wiley.com/10.1002/14651858.CD006417.pub3 